\title{
Internally generated and directly cued task sets: an investigation with fMRI
}

\author{
Birte U. Forstmann ${ }^{\mathrm{a}, *}$, Marcel Brass ${ }^{\mathrm{a}}$, Iring Koch ${ }^{\mathrm{b}}$, D. Yves von Cramon ${ }^{\mathrm{a}}$ \\ a Department of Neurology, Max Planck Institute for Human Cognitive and Brain Sciences, Leipzig, Germany \\ ${ }^{\mathrm{b}}$ Department of Psychology, Max Planck Institute for Human Cognitive and Brain Sciences, Munich, Germany
}

Received 25 February 2004; received in revised form 3 August 2004; accepted 31 August 2004

\begin{abstract}
It is widely acknowledged that the prefrontal cortex (PFC) plays a major role for goal-directed behaviour. In this context it is usually necessary to coordinate environmental information and internally represented intentions. Such goal-directed "endogenous control processes" can be investigated with the task-switching paradigm in which participants are required to alternate between different tasks. In the present study, we aimed at investigating different degrees of endogenous control by introducing two cue types with varying directness of the cue-task association. The "transition cues" informed the participants about repeating or switching the task but not about the task identity. Contrary to that, the "task cues" were directly associated with the upcoming task set. Since the transition cues are not directly associated with the task set they should require a higher demand of endogenous control than the task cues.

The comparison of both cue types revealed frontolateral as well as frontomedian activations for the transition cue. We assume that the frontolateral activation reflects the coordination of information within working memory (WM) and the frontomedian cortex reflects the higher demand for endogenous control. Furthermore, regions of interest (ROIs) analyses indicate an important role for anterior regions along the left inferior frontal sulcus and frontomedian wall. This is suggested to reflect a functional gradient in anterior-posterior direction which is linked to the relative degree of required endogenous control.
\end{abstract}

(C) 2004 Elsevier Ltd. All rights reserved.

Keywords: Prefrontal cortex; Task cues; Endogenous control

\section{Introduction}

The prefrontal cortex (PFC) has long been thought to play an important role in our ability to orchestrate thoughts and actions in accordance with the environment (Miller \& Cohen, 2001). In other words, our behaviour is governed by environmental information and internally represented information of past events and goals. Hence, in order to decide how to act in a specific situation, we usually have to integrate these external information with internally represented information. From this perspective, cognitive control can be understood as the coordination of external and internal information in working memory (WM). Whenever our behaviour is not completely externally triggered, additional internal effort is

\footnotetext{
* Corresponding author. Tel.: +49 3419940 258; fax: +49 3419940221.

E-mail address: forstman@cbs.mpg.de (B.U. Forstmann).
}

needed to which we will refer as the memory-based internal generation of task sets or endogenous control.

In the experimental literature, cognitive control has been investigated using the task-switching paradigm (Koch, 2003; Meiran, 1996; Rogers \& Monsell, 1995; Sudevan \& Taylor, 1987). In this paradigm, participants have to shift from one task to another leading to prolonged reaction times (RTs) and higher error rates which are termed "shift costs". Recently, two different task-switching procedures have been devised. One is the cueing version (see, e.g., Sudevan \& Taylor, 1987), the other one follows the alternating-runs logic with predictable task sequences (see, e.g., Rogers \& Monsell, 1995).

In cueing versions of the task-switching paradigm, an external task cue is presented which is directly, i.e. unambiguously associated with the upcoming task. This offers the opportunity to present tasks in random order because the upcoming task is reliably signalled by the external task 
cue (Sudevan \& Taylor, 1987). However, this paradigm minimises the need for endogenous control components because of the facilitative impact of the task cue on the memory-based retrieval of the relevant task set, i.e. the internal representation of the task goal, due to the direct cue-task association (Koch, 2003; Logan \& Bundesen, 2003).

In contrast to paradigms using external task cues, Rogers and Monsell (1995) introduced the alternating-runs version of the task-switching paradigm. This version does not require external task cues because simple and predictable task sequences are used, e.g. AABBAA etc. (Rogers \& Monsell, 1995). In alternating-runs versions, the integration of internal and external information is necessary in order to select the relevant task set. Hence, these designs rely on maintaining the task order in working memory to retrieve the now-relevant task set. Such studies are more valuable to make inferences about the internal generation of task sets because the task set is not exclusively triggered by a direct cue-task association.

\subsection{Neuroimaging results of the task-switching paradigm}

Several neuroimaging studies used a cueing version to investigate preparation-related activation (Brass \& von Cramon, 2002, 2004; Dove, Pollman, Schubert, Wiggins, \& von Cramon, 2000; Luks, Simpson, Feiwell, \& Miller, 2002; Ruge, Brass, Koch, Rubin, Meiran, \& von Cramon in press). Brass and von Cramon $(2002,2004)$ as well as Ruge et al. (in press) found that a frontal network involving an area in the vicinity of the left precentral sulcus and inferior frontal sulcus (the so called inferior frontal junction, IFJ) and the pre-SMA are crucial components in task preparation.

Neuroimaging studies which investigated the alternatingruns paradigm revealed a quite heterogenous data pattern. For example, Sohn, Ursu, Anderson, Stenger and Carter (2000) introduced on half of the blocks "foreknowledge", so that information about task repetition or task switch was available. A right lateralized prefrontal activation (BA 46/45) among parietal and subcortical activations was found for the foreknowledge condition. In a different study, Dreher, Koechlin, Ali and Grafman (2002) manipulated the capability for endogenous preparation by contrasting blocks in which trials alternated in a predictable manner against blocks in which the task switched randomly. Interestingly, the data revealed that the anterior medial prefrontal cortex and the left frontopolar cortex were more related to endogenous control processes, being activated when knowing which task was going to occur, while the lateral PFC was more involved with cognitive control processes in relation to external events. These findings suggest that regions more anterior situated in the prefrontal cortex come into play when the need for endogenous control is increased (Dreher et al., 2002).

Evidence for a functional gradient in anterior-posterior direction for the lateral PFC is also reported by several other authors (see, e.g., Koechlin, Ody, \& Kouneiher, 2003). The concept of such a gradient is based on the idea that activations in more anteriorly located prefrontal areas such as the middle frontal gyrus (MFG) and the frontopolar cortex tend to exhibit complex properties dependent on higher-order task processes which require the temporal integration of information (see, e.g., Fuster, 2004). In contrast, more posteriorly situated prefrontal regions such as the inferior frontal junction area reveal activation when the task set is directly triggered by external events (Brass \& von Cramon, 2004; Derrfuss, Brass, \& von Cramon, 2004).

Taken together there are several neuroimaging studies which used either the cueing version, in which the task set is externally induced through a direct task cue, or followed the alternating-runs logic, where in general the task set is not directly triggered by an external cue. It was the aim of the present study to investigate varying degrees of required endogenous control by manipulating the directness of the association between cue and task. Therefore, we devised a new cue type which we refer to as transition cues (cf. Rushworth, Hadland, Paus, \& Sipila, 2002). With this, we aimed to show neural correlates of the internal generation of task sets which requires a higher demand of endogenous control than directly cued task sets.

\subsection{Manipulating the cue-task association}

The rationale of introducing transition cues was to devise a synthesis of both the alternating-runs- and the cueing paradigm to obtain a contrast between relatively more internally generated and relatively more directly cued task sets. Therefore, two different types of external cues were used (Fig. 1A and B).

The transition cue is comprised of an indirect association between cue and task. That is, this cue type informs the participants about repeating or switching the task but not about the task identity (see, e.g., Rushworth et al., 2002). The characteristic feature of the alternating-runs paradigm, namely the need to relatively stronger internally generate the task set because of the lack of a strong direct task set information, could be realised with the transition cue type while being able to use unpredictable task sequences comparable to the task cueing procedure. Contrary to that, the task cue is comprised of a direct association between cue and task, thus being identical to the task cues used in cueing versions of previously adopted task-switching studies (see, e.g., Brass \& von Cramon, 2002; Koch, 2003). Participants had to shift between two numerical judgement tasks either deciding whether a given digit is greater or smaller than 5 (magnitude task) or whether it is odd or even (parity task). Each of the two task cues were directly associated with either the magnitude task or the parity task, respectively, whereas transition cues instructed a switch or repeat of the task, thereby requiring the participants to infer the now-relevant task identity.

The transition cue offers several methodological advantages compared to previously adopted task-switching procedures. One is concerned with the opportunity to investigate the internal generation of task sets while using an external 


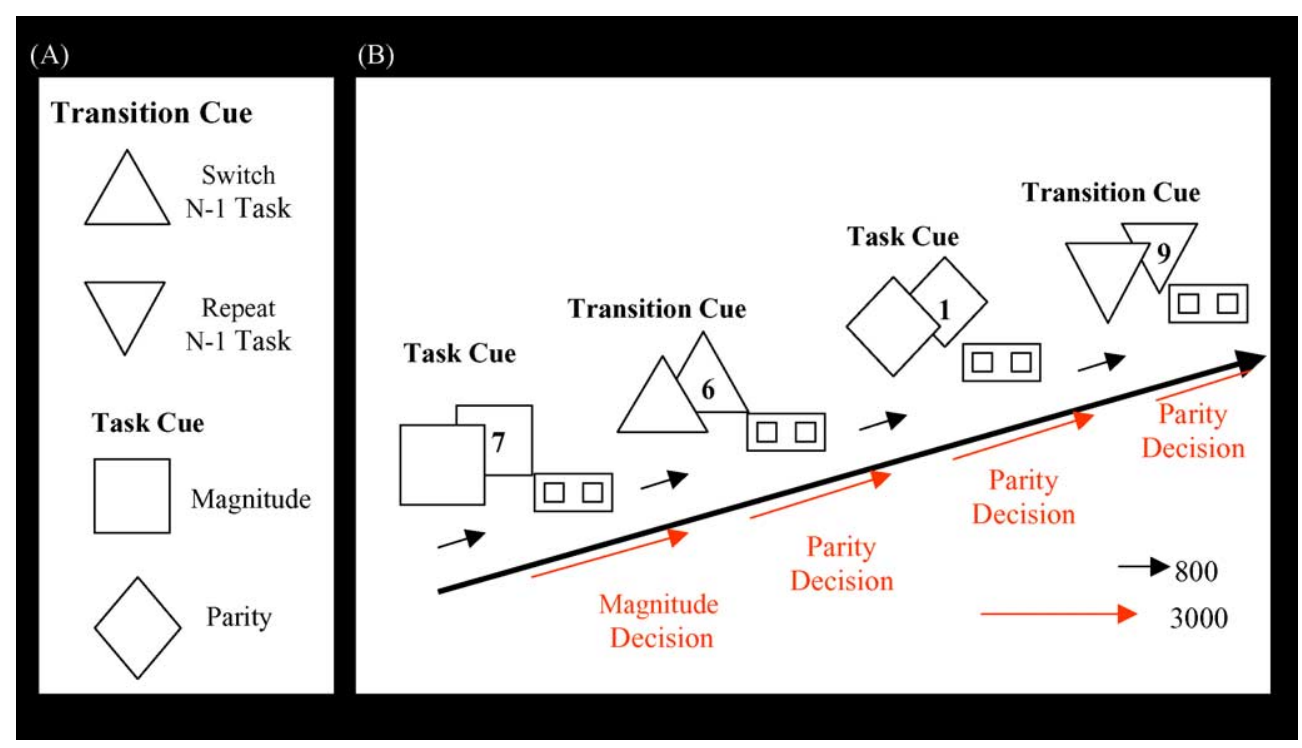

Fig. 1. Transition Cueing Paradigm. (A) Two different cue types (transition cue and task cue). The transition cue indicates whether the N-1 task should be repeated or switched whereas the task cue is directly associated with either Task A or Task B. (B) The two cue types were presented in pseudo-randomised order. A trial lasted $6000 \mathrm{~ms}$ while the cue-target interval (CTI) was held constant ( $800 \mathrm{~ms}$; black line) and the reaction time window ended after $3000 \mathrm{~ms}$ (red line).

cue type. This allows to determine the point of time when the internal generation process occurs (see Meiran, 2000a,b for a discussion). Usually, this is not possible in studies using the alternating-runs paradigm, where it might be possible to begin preparing for the required task on trial $\mathrm{N}$ before having completed the response on trial N-1. A second advantage enfolds the opportunity to adopt a design in which transition cues can be intermixed with task cues, hence allowing an event-related fMRI analysis of an intermixed design.

\section{Materials and methods}

\subsection{Participants}

Sixteen healthy volunteers were recruited. We obtained written consent from all 16 participants prior to the scanning session. All subjects had normal or corrected-to-normal vision. No subject had a history of neurological, major medical, or psychiatric disorder. The data of two subjects were excluded from the analysis due to nausea of one participant during the scanning procedure and technical problems with the headphones in the other case. The remaining 14 subjects were six females and eight males (age: mean $=23.6$, S.D. $=2.08)$ who were all right handed as assessed by the Edinburgh Inventory (Oldfield, 1971).

\subsection{Behavioural task}

Digits between 1 and 9 (except 5) were presented on the computer screen. Participants had to execute two tasks: judging whether a digit was smaller or greater than 5 (magnitude task) or judging whether a given digit was odd or even (parity task). Which task they had to execute was instructed by either a transition cue or a task cue. The transition cue consisted of (a) a triangle on its tip or (b) a triangle on its base which instructed the participants to either repeat or switch the previously performed task. The task cue was comprised of (a) a square or (b) a diamond. These were directly associated with either the parity or magnitude task (Fig. 1A).

The timing of the sequence of trials was triggered from the MRI control every $6 \mathrm{~s}$. The trials started with a variable oversampling interval of $0,500,1000$ or $1500 \mathrm{~ms}$ to obtain an interpolated temporal resolution of $500 \mathrm{~ms}$ (Miezin, Maccotta, Ollinger, Petersen, \& Buckner, 2002). Then, a fixation cross was presented for $50 \mathrm{~ms}$ followed by one of the four cues, which were always presented for $800 \mathrm{~ms}$. Thus, the cue-target interval (CTI) was held constant. While the cue remained on the screen, the digit was presented in the centre of the cue. Cue and digit together remained on the screen until a response was made or the response time interval of $3000 \mathrm{~ms}$ was exceeded. Responses were made with the right index or middle finger by pressing either the left key if a digit was smaller than 5 or odd or the right key if a digit was bigger than 5 or even (Fig. 1B).

The experiment consisted of two blocks with 160 experimental trials each, resulting in approximately 80 trials for the combinations of the two factors cue type (transition cue versus task cue) by trial type (switch versus repeat). Trials were presented in a pseudo-randomised order to equal the number of switch and repetition trials and the transition probabilities of the different conditions and cue types. We also included 40 non-events, which were pseudo-randomly interspersed. The non-events were included to compensate the overlap of the BOLD response between adjacent trials. The experiment lasted about $50 \mathrm{~min}$. Stimuli were displayed using VisuaStim (Magnetic Resonance Technologies, 
Northridge, USA), consisting of two small TFT-monitors placed directly in front of the eyes, simulating a distance to a normal computer screen of about $100 \mathrm{~cm}$ with a resolution of $1024 \times 768$ and a refresh rate of $60 \mathrm{~Hz}$. Each trial lasted $6 \mathrm{~s}$. We used a TR of $2 \mathrm{~s}$ which resulted in 540 time steps for each block. Every block started out with two dummy trials which were excluded from further analysis.

\subsection{MRI scanning procedure}

The experiment was carried out on a 3T scanner (Medspec 30/100, Bruker, Ettlingen). Twenty axial slices were acquired $(19.2 \mathrm{~cm}$ FOV, $64 \times 64$ matrix, $4 \mathrm{~mm}$ thickness, $1 \mathrm{~mm}$ spacing) parallel to the AC-PC plane and covering the whole brain. Slice gaps were interpolated to generate output data with a spatial resolution of $3 \mathrm{~mm} \times 3 \mathrm{~mm} \times 3 \mathrm{~mm}$. We used a single shot, gradient recalled EPI sequence (TR $2000 \mathrm{ms,}$ TE $30 \mathrm{~ms}, 90^{\circ}$ flip-angle). Prior to the functional runs, corresponding 20 anatomical MDEFT slices and 20 EPI-T1 slices were acquired.

\subsection{Data analysis}

\subsubsection{Preprocessing of fMRI data}

The analysis of the fMRI data was performed by using the LIPSIA software package (Lohmann et al., 2001). The functional data were first corrected for movement artefacts. Further, the temporal offset between the slices acquired in one scan were corrected by using a sinc-interpolation algorithm. Data were filtered using a spatial Gaussian filter with sigma $=0.6$. A temporal highpass filter with a cutoff frequency of $1 / 100 \mathrm{~Hz}$ was used for baseline correction of the signal. All functional data sets were individually registered into stereotactic Talairach space using the participants' individual high-resolution reference image. These 3D reference data sets were acquired during a previous scanning session. The 2D anatomical MDEFT slices, geometrically aligned with the functional slices, were used to compute a transformation matrix containing 6 degrees of freedom (three rotational, three translational) that registered the anatomical slices with the 3D reference image. These transformation matrices were normalised to the standard Talairach brain size (Talairach \& Tournoux, 1988) by linear scaling, and finally applied to the individual functional data.

\subsubsection{Statistical evaluation}

For the computation of whole-brain contrasts, we used the General Linear Model for serially autocorrelated observations (Friston, Holmes, Worsley, Poline, Frith, \& Frackowiak, 1995). The design matrix for event-related analysis was created by using a model of a synthetic BOLD function (Friston, Fletcher, Josephs, Holmes, Rugg, \& Turner, 1998). Event-onsets were synchronised with the presentation of the cue. Only correct trials were included in the analysis. The model equation, including the observation data, the design matrix, and the error term, was convolved with a Gaussian kernel with a dispersion of $4 \mathrm{~s}$ FWHM to deal with the temporal autorcorrelation (Worsley \& Friston, 1995). In the following, contrast maps, i.e. estimates of the raw-score differences between specified conditions, were generated for each participant. A one-sample Student's $t$-test of contrast maps across participants (random-effects-model considering participants as a random variable) was computed to indicate whether observed differences between specified conditions were significantly different from zero. Subsequently, $t$ values were transformed into $Z$-scores. To protect against false positive activations, only regions with a $Z$-score higher than $3.1(p<0.001$, uncorrected) and with a volume greater than $450 \mathrm{~mm}^{3}$ (10 adjacent voxel) are reported (Forman, Cohen, Fitzgerald, Eddy, Mintun, \& Noll, 1995).

Furthermore, we were interested in obtaining ROI $\times$ cue type interactions between three frontolateral and two frontomedian regions of interest (ROIs) for both cue types to show a functional gradient in anterior direction for the transition cue. The underlying signal time courses were computed for the most activated voxel of the frontopolar PFC (FPFC), the mid-MFG and IFJ on the frontolateral side for each participant. On the frontomedian side, we computed the signal time course for the most activated voxel in BA 8 and for a mean coordinate in the pre-SMA/SMA (x: 1, y: 5, z: 53). While the latter coordinate was derived from the study of Brass and von Cramon (2002), all other ROIs were determined from the random effects analysis of the main contrast between both cue types (see Table 1). On the lateral prefrontal cortex, the coordinate of the mid-MFG and the coordinate of the FPFC were chosen because it has been argued by several authors that these areas are important for the working memory related integration of information or processes called "branching", respectively (D'Esposito, Postle, Ballard, \& Lease, 1999; Fletcher \& Henson, 2001; Koechlin, Basso, Pietrini, Panzer, \& Grafman, 1999). On the medial side, BA 8 has been argued to be important for uncertainty in decision making (see, e.g., Volz, Schubotz, \& von Cramon 2003). The more posteriorly situated ROIs on the lateral and medial PFC, i.e. IFJ and pre-SMA, belong to a cortical network commonly found with explicit task cues (see, e.g., Brass, 2002; Ruge et al., in press).

The percent signal change was calculated in relation to the mean signal intensity across all timesteps. The signal change was averaged for each condition for $12 \mathrm{~s}$ after cue presentation. We then subtracted the time course of the non-event from the time course of the relevant conditions to attenuate the overlap of the BOLD response. Furthermore, we determined the maximum signal in the time window between 4 and $8 \mathrm{~s}$. Finally, the maximum signals of three lateral and two medial ROIs were submitted separately to an analysis of variance (ANOVA). For the ROI analysis, effects were considered significant at an alpha of 0.05 .

For the visualisation of a functional-anatomical gradient in the anterior-posterior direction, we masked the estimated beta values of each difference of cue type and non-event separately with the $Z$-map resulting from the main contrast (transition cue versus task cue) with a $Z$-score higher than 3.1 
Table 1

Anatomical location and Talairach coordinates for the comparison of transition cue and task cue with $Z>3.1(p=0.001$, uncorrected). Activated areas that lie $>1 \mathrm{~cm}$ apart. Activations with a minimum volume size of $450 \mathrm{~mm}^{3}$ (10 adjacent voxels) are shown

\begin{tabular}{|c|c|c|c|c|c|c|c|c|}
\hline \multirow[t]{2}{*}{ Anatomical area } & \multicolumn{4}{|c|}{ Left hemisphere } & \multicolumn{4}{|c|}{ Right hemisphere } \\
\hline & \multicolumn{3}{|c|}{ Talairach coordinates } & \multirow[t]{2}{*}{$Z_{\max }$} & \multicolumn{3}{|c|}{ Talairach coordinates } & \multirow[t]{2}{*}{$Z_{\max }$} \\
\hline \multicolumn{7}{|l|}{ Frontal } & & \\
\hline Frontopolar & & & & & 29 & 47 & 15 & 4.1 \\
\hline Inferior frontal gyrus & -52 & 14 & 9 & 4.1 & 44 & 11 & 24 & 4.0 \\
\hline Posterior middle frontal gyrus & -44 & 8 & 41 & 4.1 & & & & \\
\hline Mid-middle frontal gyrus & -44 & 23 & 27 & 4.0 & & & & \\
\hline Anterior middle frontal gyrus & -43 & 35 & 6 & 4.0 & & & & \\
\hline Inferior frontal junction & -35 & -4 & 32 & 3.9 & & & & \\
\hline Anterior insula & -29 & 17 & -3 & 3.9 & 35 & 20 & 0 & 4.7 \\
\hline Premotor cortex & & & & & 32 & 5 & 50 & 3.8 \\
\hline Frontomedian cortex (BA 8) & -5 & 17 & 50 & 3.7 & & & & \\
\hline \multicolumn{9}{|l|}{ Parietal } \\
\hline Intraparietal sulcus & -37 & -52 & 38 & 4.5 & 35 & -46 & 38 & 42 \\
\hline Supramarginal gyrus & -46 & -46 & 44 & 4.5 & 43 & -43 & 47 & 3.9 \\
\hline Upper precuneus & & & & & 8 & -70 & 47 & 4.2 \\
\hline \multicolumn{9}{|l|}{ Subcortical } \\
\hline Thalamus & & & & & 11 & -10 & 6 & 3.7 \\
\hline
\end{tabular}

( $p<0.001$, uncorrected). This assured us that only voxels being significantly activated in the random effects analysis for the main contrast of cue type were chosen except for the coordinate in the pre-SMA which was derived from the study from Brass and von Cramon (2002).

\section{Results}

\subsection{Behavioural performances}

The behavioural results (Fig. 2A and B) revealed a significant main effect for the cue type, $F(1,13)=56.6 ; p<0.001$, indicating that RTs for the transition cue are higher than for the task cue (1124 ms versus $907 \mathrm{~ms}$ ). In addition, the main effect for trial type was significant, $F(1,13)=25.38 ; p<0.001$, indicating that RTs in switch trials were higher than for nonswitch trials ( $1060 \mathrm{~ms}$ versus $971 \mathrm{~ms}$ ). The interaction of cue type and trial type was significant, $F(1,13)=6.00 ; p<0.05$, indicating higher switch costs for the transition cue in switch trials compared to the task cues ( $138 \mathrm{~ms}$ versus $40 \mathrm{~ms}$ ).

The overall accuracy was $94.7 \%$. Error rates were submitted to the same analysis as RTs. The ANOVA revealed a significant main effect for cue type, $F(1,13)=31.08$; $p<0.01$, showing higher error rates for the transition cues (8\%) compared to the task cues $(3 \%)$. No further main effect or interaction reached significance $(F<1)$. Thus, no speed-accuracy trade-off can be made responsible to account for the RT data pattern.

\subsection{Neuroimaging data}

The first question we addressed in the analysis of the fMRI data was whether brain activation associated with the internal generation of task sets would significantly differ from directly cued task sets. The random effects analysis for the main contrast of transition cues versus task cues revealed several activation foci along the left inferior frontal sulcus (Table 1 and Fig. 3). There was an activation on the mid-part of the left middle frontal gyrus (mid-MFG) which extended in posterior and anterior direction of the middle frontal gyrus (posterior MFG and anterior MFG) and reached the IFJ. Furthermore, bilateral activation was found in the posterior part of the inferior frontal gyrus, namely at the tip of Broca's area (BA 44). The frontopolar PFC (FPFC) as well as the ven-
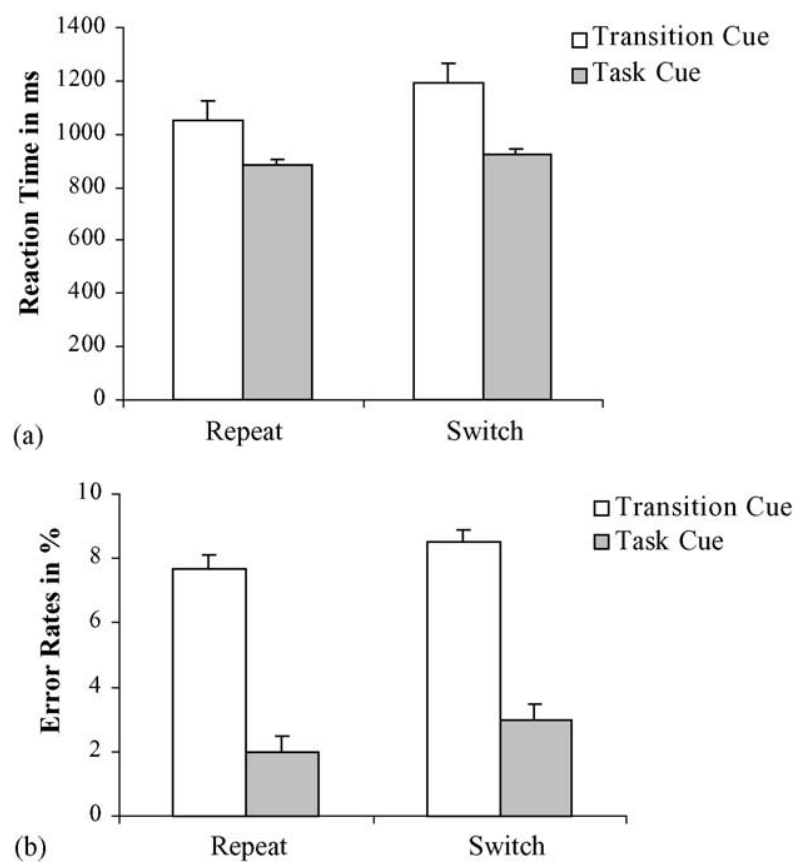

Fig. 2. (A) Mean RT in ms as a function of cue type (transition cue vs. task cue) and trial type (switch vs. repeat). (B) Mean error rates in \% as a function of cue type (transition cue vs. task cue) and trial type (switch vs. repeat). 


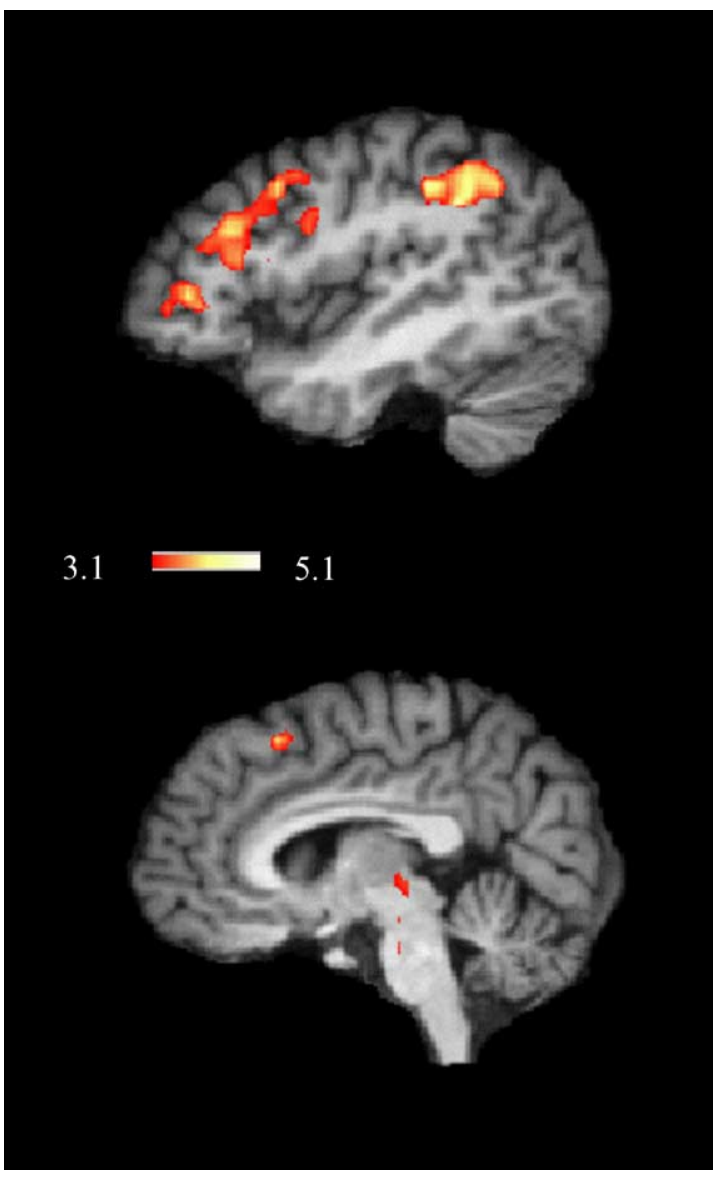

Fig. 3. Activation map averaged over all 14 subjects ( $Z$ threshold at $Z=3.1$ ) mapped onto the mean brain of all subjects. Shown is the sagittal view of the left lateral cortex $(x=-43)$ and the frontomedian wall $(x=-5)$ for the main contrast for the transition cue vs. task cue. Red labels indicate positive $Z$ values.

tral premotor cortex revealed exclusive activation in the right hemisphere. Medially, activation was found in the dorsal part of BA 8 extending into the pre-SMA. In the following sections we will refer to this activation as medial BA 8. Furthermore, the anterior insula was activated bilaterally. The parietal lobe revealed activation along the ascending branch of the intraparietal sulcus (IPS), in the supramarginal gyrus bilaterally and in the upper precuneus (BA $7 \mathrm{~m}$ ) of the right hemisphere. Finally, the right thalamus was significantly activated.

A further analysis was concerned with frontal areas subserving the (re)-configuration of task sets, namely the areas elicited in switch trials. Therefore, we calculated the main effect of trial type (switch versus repeat) applying the defined threshold criteria derived from the main effect of cue type. The results revealed no significantly activated prefrontal areas for the main effect of trial type. However, we obtained parietal and subcortical activations which will not be further addressed. The results of the whole-brain interaction between cue type and trial type revealed also no prefrontal activation. Hence, in all subsequently following analyses, we focused on activations obtained from the whole-brain contrast between both cue types.
The second question of this study was concerned with a functional gradient in anterior-posterior direction for the transition cue compared to the task cue. Therefore we obtained ROI $\times$ cue type interactions for three lateral prefrontal ROIs (FPFC, mid-MFG and IFJ) and both cue types (transition cue versus task cue; see also Fig. 4) as well as two frontomedian ROIs (BA 8 and pre-SMA) and both cue types, respectively. The results revealed a significant interaction between the frontolateral ROIs $\times$ cue type, $F(2,12)=7.36$; $p<0.01$. In post hoc analyses, we then calculated the percent signal change difference between transition cues and task cues for each lateral ROI and adopted three two-sided Student's $t$-tests with effects to be considered significant at an alpha of 0.05 . The results revealed a significant difference between mid-MFG and IFJ, $t(13)=-3.79, p=0.002)$, indicating a larger cue type effect for the mid-MFG compared to the IFJ. All other lateral ROI $\times$ cue type effects were not significant $(p>0.1)$. For the frontomedian wall, the ROI $\times$ cue type interaction revealed significance, $F(1,13)=9.11 ; p<0.01$, indicating a larger percent signal change difference in BA 8 compared to the percent signal change in the pre-SMA for the transition cue compared to the task cue. In Fig. 4, the activation shift on the lateral PFC along the left inferior frontal sulcus and medial PFC is shown by the estimated beta values for each cue type which are higher than 0.2. Note that the mean coordinate in the pre-SMA derived from the study of Brass and von Cramon (2002) revealed no difference between both cue types for the estimated beta values which is consistent with the ROI analysis.

\section{Discussion}

The present study aimed at investigating neural correlates of endogenous control when the task set is not directly cued. Previous task-switching studies used either predictable task sequences or they relied on task cues that are directly associated with the task set. Here, we investigated brain regions subserving the internal generation of task sets by contrasting transition cues which exhibit an indirect cue-task association with task cues which exhibit a direct cue-task association.

Several frontolateral activations and one frontomedian activation were found to be more strongly activated for the transition cue compared to the task cue. When comparing the present results with previous findings from task-cueing paradigms, it becomes evident that the pattern of the frontal activations usually found with task cues shifted in an anterior direction (see, e.g., Brass \& von Cramon, 2002, 2004; Ruge et al., in press) on both the frontolateral cortex as well as the frontomedian wall. ROI $\times$ cue type interactions corroborated this finding.

The contrast between switch and repetition trials as well as for the interaction between cue type and trial type revealed no frontal activations. This finding is in accordance with a number of recent studies which failed to find stronger frontal activations for the contrast of switch and repeat trials with a 


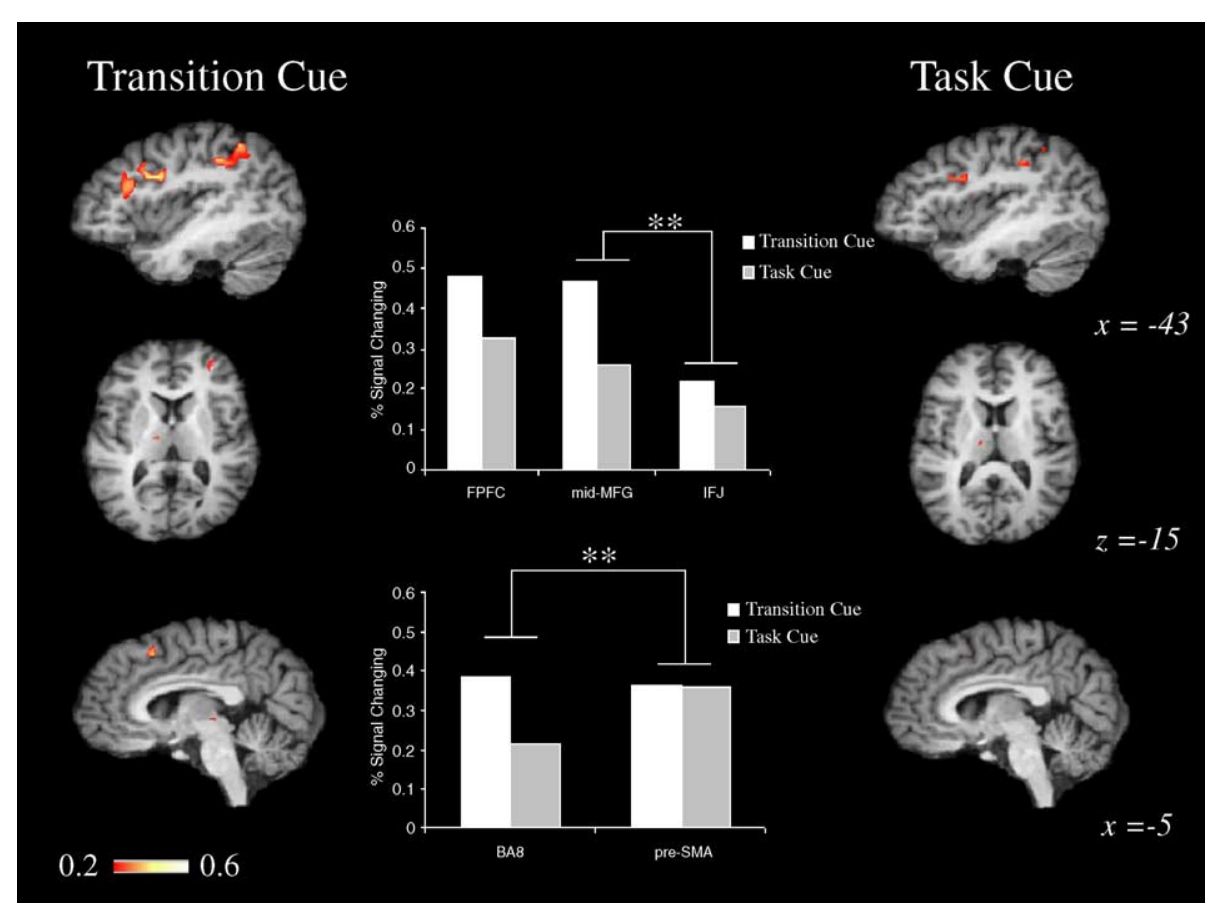

Fig. 4. Separately masked estimated beta values for each random effects analysis of cue type and non-event (i.e., transition cue vs. non-event and task cue vs. non-event) with the random effects analysis of the main contrast (transition cue vs. task cue) with a $Z$-score higher than 3.1 ( $p<0.001$, uncorrected). Only voxels being significantly activated and with a beta value higher than 0.2 are displayed. Red labels indicate beta values ranging from 0.2 to 0.6 . Diagrams report the averaged percent signal change for both cue types (transition cue and task cue) for three frontolateral ROIs (FPFC $x$ : 29, $y: 47, z: 15$; mid-MFG $x$ : $-44, y$ : 23, $z: 27$; IFJ $x:-35, y:-4, z: 32$ ) and two frontomedian ROIs (BA $8 x:-5, y: 17, z: 50$; pre-SMA $x: 1 ; y: 5 ; z: 53$ ). Asterisks indicate the significant ROI $\times$ cue type interaction between two left lateral ROIs (mid-MFG and IFJ) and two frontomedian ROIs (BA 8 and pre-SMA) with the two cue types, respectively.

long CTI (Brass \& von Cramon, 2002, 2004; Ruge et al., in press). They argued that the necessity and demand of exerted cognitive control might be equivalent for both trial types. This is especially the case for an experimental context in which there is an equal likelihood of switch and repetition trials. Therefore, the contrast between trial types may not be a suitable indicator of cognitive control processes (Brass \& von Cramon, 2002, 2004).

\subsection{The role of the lateral prefrontal cortex for internally generated and directly cued task sets}

In the present study, several frontolateral activations were obtained for the transition cue compared to the task cue. Among them is the left inferior frontal junction, the left midMFG extending along the inferior frontal sulcus, and the right frontopolar PFC which have been observed in a number of task-switching studies using both predictable task sequences or direct task cues (Brass \& von Cramon, 2002, 2004; Dreher et al., 2002; Luks, Simpson, Feiwell, \& Miller, 2002; Ruge et al., in press; Sohn et al., 2001).

Several studies implicate that the IFJ is relevant for context-related updating of general task representations, which has mainly emerged from task-switching studies using direct task cues (Brass \& von Cramon, 2002, 2004; Dove et al., 2000; Sylvester et al., 2003) and Wisconsin Card Sorting studies (Monchi, Petrides, Petre, Worsley, \& Dagher 2001; Nagahama et al., 2001).
Activations along the inferior frontal sulcus, especially the mid-MFG have been shown by a variety of tasks where a high degree of cognitive control was required in order to "monitor", "maintain", and "integrate" information over a period of time (D'Esposito et al., 1999; Fletcher \& Henson, 2001; Frith \& Dolan, 1996; Owen et al., 1999). With the transition cue, differential sources of contextual information ( $\mathrm{N}-1$ task set) and the present cue information have to be integrated. Therefore, we suggest that the left frontolateral activations reflect the integration of information within working memory.

Additionally, the right FPFC revealed higher activation for the transition cue compared to the task cue. The FPFC is said to be associated with a process that has been termed "branching" (see also Koechlin et al., 1999). Branching refers to the holding of a primary task in working memory while a secondary task has to be carried out. This applies to the requirements of the transition cue since the participants have to internally generate the task set while coordinating the contextual information within working memory.

\subsection{The role of the medial-frontal wall for internally generated and directly cued task sets}

The comparison of activations of both cue types revealed an activation peak in the medial BA 8 for the transition cues. Contrary to that, previous task-switching studies revealed that the pre-SMA/SMA is involved in cue-related processing (Brass \& von Cramon, 2002; Dove et al., 2000; Luks 
et al., 2002; Rushworth et al., 2002). Furthermore, the preSMA is assumed to be important for the internal generation and preparation of motor activity (Lee, Chang, \& Roh, 1999; Picard \& Strick, 1996, 2003). Regarding the present findings, it is conceivable that a functional gradient from the pre-SMA into the rostrally situated BA 8 is elicited by the indirect cue-task associations in which the external transition cue information does not determine the relevant task set. Evidence for the account of a functional gradient in anterior direction was also revealed by a ROI $\times$ cue type interaction of the medial BA 8 and a coordinate in the pre-SMA. Here we could show that the medial BA 8 compared to the pre-SMA plays a dominant role for the transition cue compared to the task cue.

However, it is necessary to specify the subprocesses being relevant in indirect cue-task associations. Besides the memory aspect which requires the participants to integrate information over a period of time, it is necessary to internally generate the now-relevant task set representation due to the indirect cue-task association.

We presume that the medial BA 8 is subserving this internally driven process. This is further corroborated by studies investigating the internal generation of synonyms (Nyberg et al., 2003), uncertainty in decision making (Volz, Schubotz, \& von Cramon, 2003) as well as during rule application (Goel \& Dolan, 2000), serial event prediction in increasingly complex stimulus trains (Schubotz \& von Cramon, 2002), and response competition (Ullsperger and von Cramon, 2004).

Finally we suggest that the medial BA 8 is important for the internal generation of task sets which becomes relevant for solving environmental ambiguity. This is in accordance with the view that less determined action contexts require a higher demand of endogenous control compared to directly cued task sets.

\subsection{A functional gradient in the anterior-posterior dimension along the lateral and medial PFC}

Recently, several authors proposed a functional-anatomical gradient in the lateral PFC along an anterior-posterior dimension (Braver, Reynolds, \& Donaldson, 2003; Christoff \& Gabrieli, 2000; Dreher et al., 2002; Koechlin et al., 2003); Reynolds \& Donaldson, 2003). Evidence for a cytoarchitectonic gradient relies inter alia on studies conducted by Sanides (1962). However the functional characterisation of such a gradient remained open. Braver et al. (2003) suggested that the PFC might be organised according to the temporal duration of actively maintained representations, with the most anterior regions being recruited under conditions where active memory needs to be sustained.

Regarding the task-switching literature, Dreher et al. (2002) proposed an anterior-posterior axis as the task becomes more endogenously guided. In their study, anterior PFC activity was associated with improved performance under conditions where sustained endogenous preparation for task-switching could be achieved (i.e. when task switches were predictable across the block). Very recently, Koechlin et al. (2003) put forward a cascade model of executive processes from premotor to anterior PFC regions that control behaviour according to the stimuli, the present perceptual context, and the temporal episode in which stimuli occur, respectively.

In the present study, the results indicate a dominant role of the more anterior situated ROI on the left frontolateral cortex, namely the mid-MFG for the integration of information in working memory compared to the directly cued task sets. Furthermore, a comparable dominant role of the anterior situated ROI in the medial wall (BA 8) was obtained which is suggested to reflect the internal generation of the task set (Fig. 4). These findings lead us to suggest a functional gradient in anterior-posterior dimension for the left lateral and presumably medial PFC reflecting a higher demand of endogenous control. This holds for both the coordination of information over a certain time interval as well as for the internal generation of task sets in less determined action contexts.

\subsection{Other frontolateral activations revealed by the transition cue}

Besides the activations along the dorsal bank of the inferior frontal sulcus, we also obtained left-sided activation in the posterior part of the inferior frontal gyrus, namely the tip of Broca (BA 44). Even if we had no a-priori hypothesis for the activation in BA 44, we assume that it might reflect the phonological rehearsal of the contextual information facilitating the coordination process within working memory (Fletcher \& Henson, 2001).

\section{Conclusion}

In the present experiment, we contrasted two different types of cues to investigate cognitive control processes. The results revealed a functional gradient in anterior-posterior direction along both the left frontolateral and frontomedian cortex when comparing an indirect with a direct cue-task association. Furthermore, ROI $\times$ cue type interactions showed that the mid-MFG and the medial BA 8 play essential roles when the action context is less determined. We suggest that the activation in the mid-MFG subserves the integration of external information and internally represented information in working memory revealing a higher demand of cognitive control compared to directly cued task set representations. The activation in the medial BA 8 is associated with the requirement to internally generate the task set representation reflecting an act of endogenous control. In everyday life choosing between different courses of actions usually requires the integration of environmental information and internally represented information of past events. In this sense, working memory and endogenous control might reflect two sides of the same coin. 


\section{Acknowledgements}

We would like to thank Stefan Zysset for improvements of the fMRI analysis and Jan Derrfuss for helpful discussions. Furthermore, the authors thank two anonymous reviewers for helpful comments on the previous version of the manuscript.

\section{References}

Brass, M., \& von Cramon, D. Y. (2002). The role of the frontal cortex in task preparation. Cerebral Cortex, 12, 908-914.

Brass, M., \& von Cramon, D. Y. (2004). Decomposing components of task preparation with functional MRI. Journal of Cognitive Neuroscience, 16, 609-620.

Braver, T. S., Reynolds, J. R., \& Donaldson, D. I. (2003). Neural mechanisms of transient and sustained cognitive control during task switching. Neuron, 39, 713-726.

Buckner, R. L. (2003). Functional-anatomic correlates of control processes in memory. Journal of Neuroscience, 23, 3999-4004.

Christoff, K., \& Gabrieli, J. D. E. (2000). The frontopolar cortex and human cognition: Evidence for a rostorcaudal hierarchical organization within the human prefrontal cortex. Psychobiology, 28, 168-186.

D’Esposito, M., Postle, B. R., Ballard, D., \& Lease, J. (1999). Maintenance versus manipulation of information held in working memory: An event-related fMRI study. Brain and Cognition, 41, 66-86.

Derrfuss, J., Brass, M., \& von Cramon (in press). Cognitive control in the posterior frontolateral cortex: Evidence from common activations in task coordination, interference control, and working memory. Neuroimage.

Dove, A., Pollmann, S., Schubert, T., Wiggins, C. J., \& von Cramon, D. Y. (2000). Prefrontal cortex activation in task switching: An eventrelated fMRI study. Cognitive Brain Research, 9, 103-109.

Dreher, J. C., Koechlin, E., Ali, S. O., \& Grafman, J. (2002). The roles of timing and task order during task switching. Neuroimage, 17, 95-109.

Fletcher, P. C., \& Henson, R. N. A. (2001). Frontal lobes and human memory-Insights from functional neuroimaging. Brain, 124, 849-881.

Forman, S. D., Cohen, J. D., Fitzgerald, M., Eddy, W. F., Mintun, M. A., \& Noll, D. C. (1995). Improved assessment of signficant activation in functional magnetic resonance imaging (fMRI): Use of a cluster-size threshold. Magnetic Resonance in Medicine, 33, 636-647.

Friston, K. J., Fletcher, P., Josephs, O., Holmes, A., Rugg, M., \& Turner, R. (1998). Event-related fMRI: Characterizing differential responses. Neuroimage, 7, 30-40.

Friston, K. J., Holmes, A. P., Worsley, K. J., Poline, J. P., Frith, C. D., \& Frackowiak, R. S. J. (1995). Statistical parametric maps in functional imaging: A general linear approach. Human Brain Mapping, 2, 189-210.

Frith, C., \& Dolan, R. (1996). The role of the prefrontal cortex in higher cognitive functions. Cognitive Brain Research, 5, 175-181.

Fuster, J. (2004). Upper processing stages of the perception-action cycle. Trends in Cognitive Sciences, 8, 143-145.

Goel, V., \& Dolan, R. J. (2000). Anatomical segregation of component processes in an inductive inference task. Journal of Cognitive Neuroscience, 12, 110-119.

Koch, I. (2003). The role of external cues for endogenous advance reconfiguration in task switching. Psychonomic Bulletin \& Review, 10, $488-492$.

Koch, I. (in press). Sequential task predictability in task switching. Psychonomic Bulletin \& Review.

Koechlin, E., Basso, G., Pietrini, P., Panzer, S., \& Grafman, J. (1999). The role of the anterior prefrontal cortex in human cognition. Nature, 399, 148-151.

Koechlin, E., Ody, C., \& Kouneiher, F. (2003). The architecture of cognitive control in the human prefrontal cortex. Science, 302, 1181-1185.
Konishi, S., Hayashi, T., Uchida, I., Kikyo, H., Takahashi, E., \& Miyashita, Y. (2002). Hemispheric asymmetry in human lateral prefrontal cortex during cognitive set shifting. Proceedings of the National Academy of Sciences of the United States of America, 9, 7803-7908.

Lee, K. -M., Chang, K. -H., \& Roh, J. -K. (1999). Subregions within the supplementary motor area activated at different stages of movement preparation and execution. Neuroimage, 9, 117-123.

Logan, G. D., \& Bundesen, C. (2003). Clever homunculus: Is there an endogenous act of control in the explicit task-cuing procedure? Journal of Experimental Psychology: Human Perception \& Performance, 29, 575-599.

Lohmann, G., Mueller, K., Bosch, V., Mentzel, H., Hessler, S., Chen, L., et al. (2001). Lipsia-a new software system for the evaluation of functional magnetic resonance images of the human brain. Computing Medical Imaging Graph, 25, 449-457.

Luks, T. L., Simpson, G. V., Feiwell, R. J., \& Miller, W. J. (2002). Evidence for anterior cingulate cortex involvement in monitoring preparatory attentional set. Neuroimage, 17, 792-802.

Meiran, N. (1996). Reconfiguration of processing mode prior to task performance. Journal of Experimental Psychology, 22, 1423-1442.

Meiran, N. (2000a). Modeling cognitive control in task-switching. Psychological Research, 63, 234-249.

Meiran, N. (2000b). Reconfiguration of stimulus task sets and response task sets during task switching. In S. Monsell \& J. Driver (Eds.), Attention and Performance XVIII: Control of cognitive processes (pp. 377-399). Cambridge, MA: MIT Press.

Miezin, F. M., Maccotta, L., Ollinger, J., Petersen, M., \& Buckner, S. E. (2000). Characterizing the hemodynamic response: Effects of presentation rate, sampling procedure, and the possibility of ordering brain activity based on relative timing. Neuroimage, 11, 735-759.

Miller, E. K., \& Cohen, J. D. (2001). An integrative theory of prefrontal cortex function. Annual Reviews in Neuroscience, 24, 167-202.

Monchi, O., Petrides, M., Petre, V., Worsley, K., \& Dagher, A. (2001). Wisconsin card sorting revisited: Distinct neural circuits participating in different stages of the task identified by event-related functional magnetic resonance imaging. Journal of Neuroscience, 21, 7722-7741.

Nagahama, Y., Okada, T., Katsumi, Y., Hayashi, T., Yamauchi, H., Oyanagi, C., et al. (2001). Dissociable mechanisms of attentional control within the human prefrontal cortex. Cerebral Cortex, 11, 85-92.

Nyberg, L., Marklund, P., Persson, J., Cabeza, R., Forkstam, C., Petersson, K. M., et al. (2003). Common prefrontal activations during working memory, episodic memory, and semantic memory. Neuropsychologia, 41, 371-377.

Oldfield, R. C. (1971). The assessment and analysis of handedness: The Edinburgh Inventory. Neuropsychologia, 9, 97-113.

Owen, A. M., Herrod, H. J., Menon, D. K., Clark, J. C., Downey, S. P. M. J., Carpenter, T. A., et al. (1999). Redefining the functional organization of working memory processes within human lateral prefrontal cortex. European Journal of Neuroscience, 11, 567-574.

Picard, N., \& Strick, P. L. (1996). Motor areas of the medial wall: A review of their location and functional activation. Cerebral Cortex, 6 , 342-353.

Picard, N., \& Strick, P. L. (2003). Activation of the supplementary motor area (SMA) during performance of visually guided movements. Cerebral Cortex, 13, 977-986.

Rogers, R. D., \& Monsell, S. (1995). Costs of a predictable switch between simple cognitive tasks. Journal of Experimental Psychology, 124, 207-231.

Ruge, H., Brass, M., Koch, I., Rubin, O., Meiran, N., \& von Cramon, D. Y. (in press). Reconciling advance preparation with proactive interference in task switching: Insights from BOLD fMRI. Neuropsychologia.

Rushworth, M. F., Hadland, K. A., Paus, T., \& Sipila, P. K. (2002). Role of the human medial frontal cortex in task switching: A combined fMRI and TMS study. Journal of Neurophysiology, 87, 2577-2592.

Sanides, F. (1962). Die Architektonik des menschlichen Stirnhirns. Berlin: Springer Verlag. 
Schubotz, R. I., \& von Cramon, D. Y. (2002). Predicting perceptual events activates corresponding motor schemes in lateral premotor cortex: An fMRI study. Neuroimage, 15, 787-796.

Sohn, M. H., Ursu, S., Anderson, J. R., Stenger, V. A., \& Carter, C. S. (2000). The role of prefrontal cortex and posterior parietal cortex in task-switching. Proceedings of the National Academy of Sciences of the USA, 97, 13448-13453.

Sudevan, P., \& Taylor, D. A. (1987). The cuing and priming of cognitive operations. Journal of Experimental Psychology: Human Perception and Performance, 13, 89-103.

Sylvester, C. -Y. C., Wager, T. D., Lacey, S. C., Hernandez, L., Nichols, T. E., Smith, E. E., et al. (2003). Switching attention and resolving in- terference: fMRI measures of executive functions. Neuropsychologia, 41, 357-370.

Talairach, J. \& Tournoux, P. (1988). Co-planar stereotaxic atlas of the human brain. New York: Thieme.

Ullsperger, M., \& von Cramon, D. Y. (2004). Neuroimage of performance monitoring: Error detection and beyond. Cortex, 40, 593604.

Volz, K. G., Schubotz, R. I., \& von Cramon, D. Y. (2003). Predicting events of varying probability: Uncertainty investigated by fMRI. Neuroimage, 19, 271-280.

Worsley, K. J., \& Friston, K. J. (1995). Analysis of fMRI time-series revisited-again. Neuroimage, 2, 173-181. 